This item was submitted to Loughborough's Research Repository by the author.

Items in Figshare are protected by copyright, with all rights reserved, unless otherwise indicated.

\title{
Laser tracking system for sports ball trajectory measurement
}

PLEASE CITE THE PUBLISHED VERSION

http://dx.doi.org/10.1243/17543371JSET67

PUBLISHER

Professional Engineering Publishing / @ IMECHE

VERSION

VoR (Version of Record)

LICENCE

CC BY-NC-ND 4.0

REPOSITORY RECORD

Ronkainen, Jouni A., and Andy R. Harland. 2019. "Laser Tracking System for Sports Ball Trajectory Measurement”. figshare. https://hdl.handle.net/2134/6592. 
This item was submitted to Loughborough's Institutional Repository (https://dspace.lboro.ac.uk/) by the author and is made available under the following Creative Commons Licence conditions.

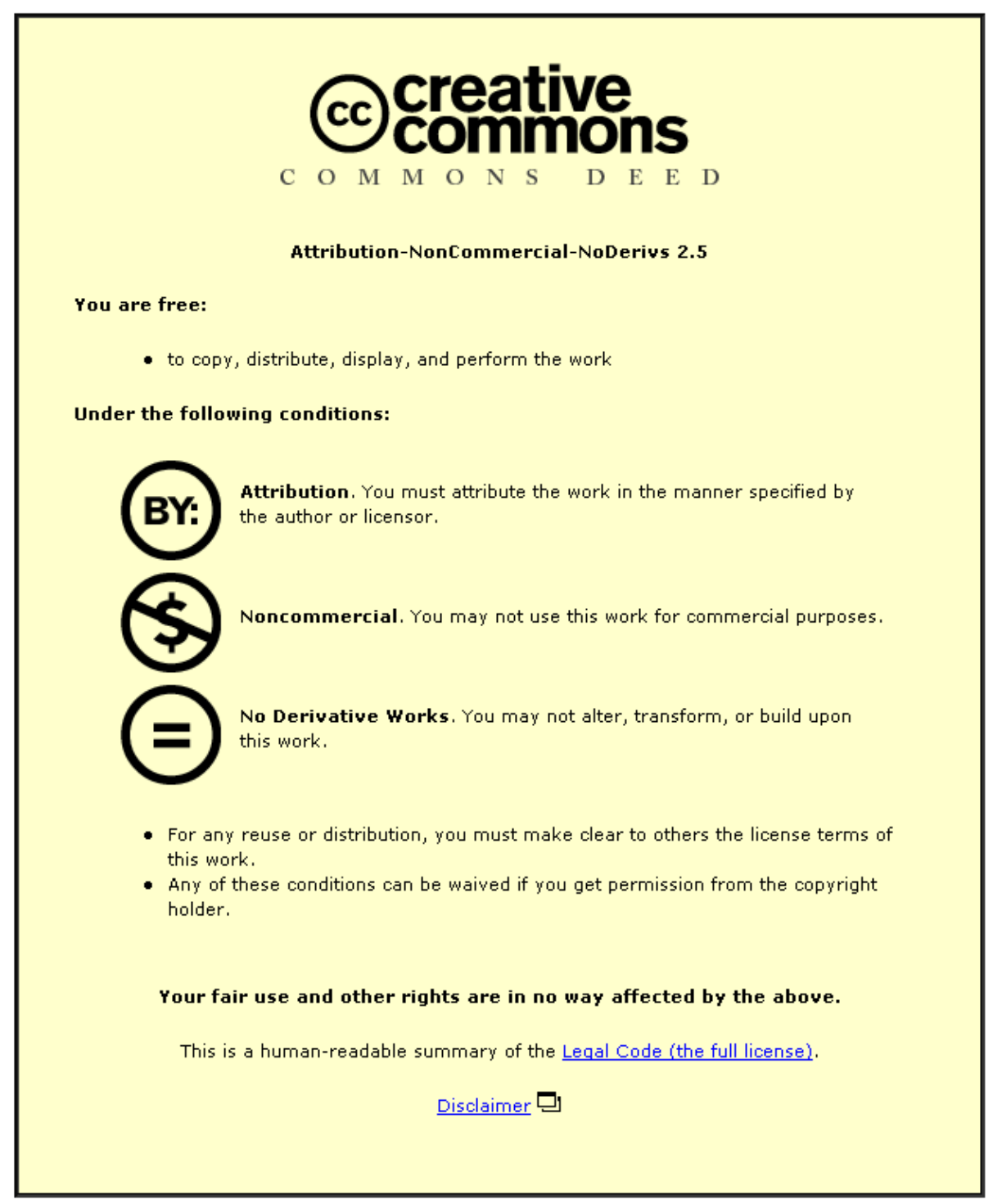

For the full text of this licence, please go to: http://creativecommons.org/licenses/by-nc-nd/2.5/ 


\title{
Laser tracking system for sports ball trajectory measurement
}

\author{
J Ronkainen and A Harland* \\ Sports Technology Institute, Wolfson School of Mechanical and Manufacturing Engineering, Loughborough \\ University, Loughborough, UK
}

The manuscript was received on 9 December 2009 and was accepted after revision for publication on 22 April 2010.

DOI: $10.1243 / 17543371$ JSET67

\begin{abstract}
The sports ball market is a multi-million dollar industry; original equipment manufacturers are continually trying to develop sports balls and in order to achieve improvement in performance they often utilize launch and/or flight monitors to allow consistent measurement and benchmarking of their balls under test. This paper introduces a laser tracking system (LTS), which has been specifically designed for soccer ball tracking. This LTS combines two different concepts together in an innovative optical solution to allow the system to track an arbitrarily moving soccer ball in a completely non-contact and non-marking methodology, tracking a ball in real time up to approximately $10 \mathrm{~m} / \mathrm{s}$. The system was programmed to act as a launch monitor capable of reporting the ball launch velocity and launch angle. In order to achieve the fully functional LTS, the hardware and software choices are clearly outlined, as well as describing the tracking methodology used to achieve the real-time tracking capability.
\end{abstract}

Keywords: soccer, high-speed video, laser tracking system, non-marking, ball trajectory, real-time tracking

\section{INTRODUCTION}

The sports ball market is extremely competitive and, in the USA alone, valued in excess of US $\$ 1305$ million [1]. Original equipment manufacturers are continually trying to create a competitive edge over their rivals. In order to understand better the ball flight and impact characteristics, many manufacturers are concerned with the quantitative measurement of launch and flight characteristics. Consequently, a range of launch and flight monitors have been developed [2-6].

Despite being heavily utilized within golf, microwave-based radar devices $[4,6]$ have not gained widespread usage in other sports. This has probably been inhibited by the large cost and complexity of operating successfully indoors because the microwaves reflect, refract, and transmit through multiple surfaces. One of the most advanced commercial

*Corresponding author: Sports Technology Institute, Wolfson School of Mechanical and Manufacturing Engineering, Loughborough University, 1 Oakwood Drive, Loughborough, Leicestershire LE11 3TU, UK.

email:A.R.Harland@lboro.ac.uk flight measurement devices based on multiple cameras [3] has been extremely successful in cricket and tennis; however, it is expensive to install, requires multiple cameras to see the ball simultaneously, and needs a permanent installation of the cameras. A purpose-built soccer ball launch measurements system [5] based on one camera capturing two sequential images of a marked ball was commercialized but was not successful in penetrating the soccer market. The main reasons for this can be attributed to the changing ambient light and background conditions in the captured images and the need to mark the soccer ball. Therefore, this device was seen as a more cost-efficient and portable solution than the other devices, but lacking in effectiveness.

The properties of laser light make it an ideal tool for accurate, non-contact, and non-marking measurements. In this paper, optical laser techniques are presented which allow measurement of the ball launch angle and velocity. In order to achieve this successfully, a system that is capable of accurately steering the laser beam to the desired target is developed. This paper reports a laser tracking system (LTS) platform. 


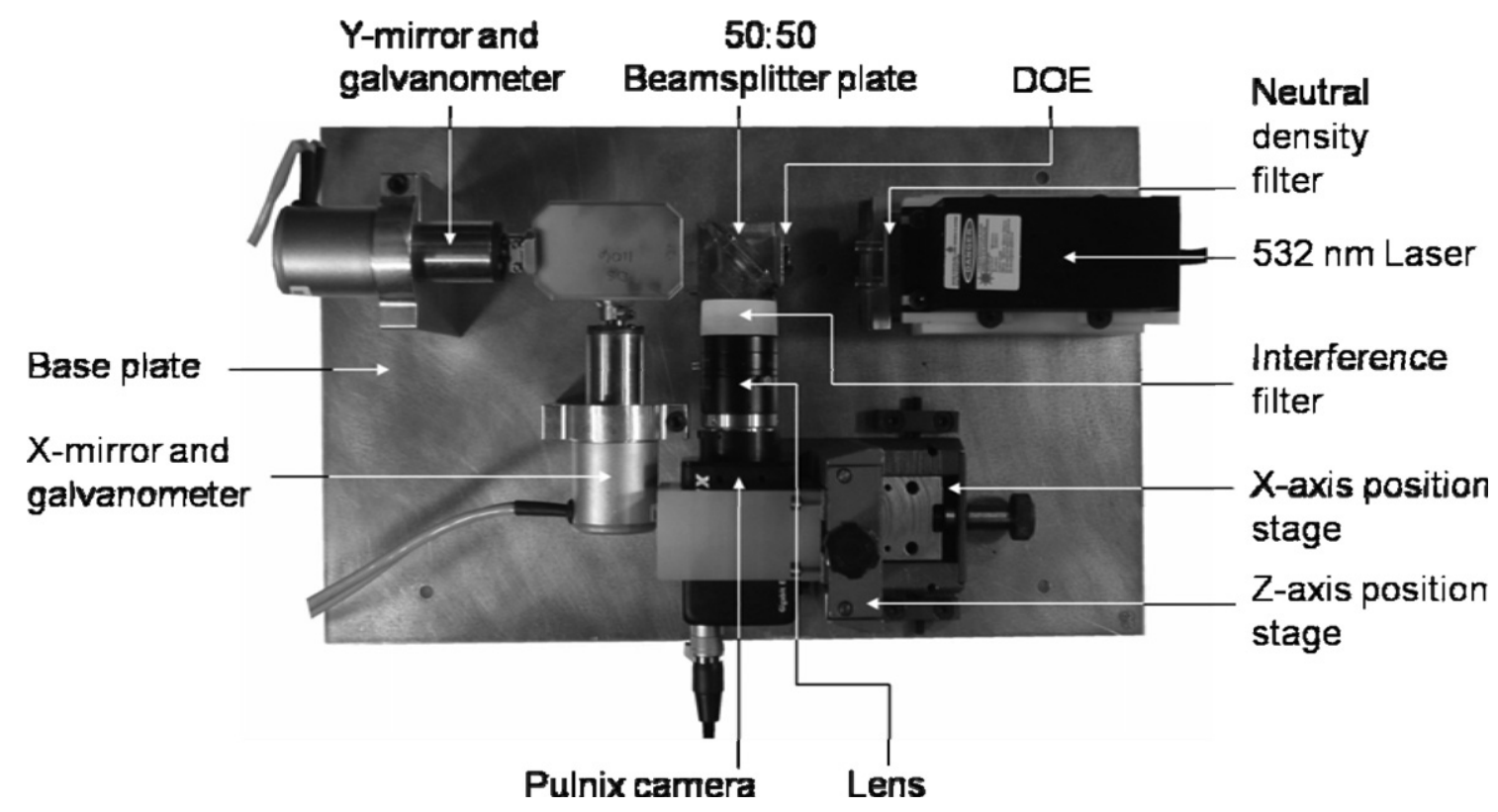

Fig. 1 Plan view of core laser tracking system components

A number of optical metrology systems have incorporated mirrors or galvanometers to direct the incident laser beam on to the target of interest, dating back to 1981 when Durst et al. [7] oscillated a mirror to redirect the path of a vibrometer laser beam. Since then, Stanbridge and Ewins [8] were the first to scan the laser beam in a circular pattern using two orthogonally aligned galvanometers, and Castellini and Tomasini [9] furthered this technique by creating an image-based tracking laser Doppler vibrometer system.

Apart from laser vibrometry, optical techniques for tracking targets have also been developed, most notably by Perrin and co-workers [10-12], who reported tracking human finger movement as a noncontact approach to interacting with computers and/or hand-held devices. In their work, human gestures were reported to be tracked at velocities less than $3 \mathrm{~m} / \mathrm{s}$.

\subsection{LTS concept design and description}

The concept of the work by Castellini and Tomasini [9] whereby a single-point vibrometer and high-speed camera were aligned to have identical optical axes was taken as a premise for this work. The tracking methodology was based on the concept used by Perrin et al. $[10,11]$ whereby movement of the target within the field of view (FOV) was monitored and mitigated through the repositioning of two orthogonal mirrors in a continuous feedback loop. The combination of these two principles realized within a novel methodology has led to the development of the LTS. The hardware and software choices are outlined in order to justify the final components for the fully functional prototype system.

\subsection{Hardware}

In order to create the LTS, careful attention was given to the implemented hardware. The system as a whole required that components complemented one another; therefore, components were not selected in isolation. The core LTS components are shown in Fig. 1.

\subsubsection{The camera}

A 32 bit peripheral component interconnect (PCI) bus architecture personal computer (PC) was used to execute bespoke tracking software. The maximum bandwidth for data transfer was therefore limited to $132 \mathrm{Mb} / \mathrm{s}$ [13]. A gigabit Ethernet (GigE) PC interface was selected, which permitted the fastest available connection without the need for a frame grabber card [14].

A global review of GigE cameras was carried out by Wilson [15]. The ideal camera based on a high frame rate, combined with a large pixel resolution, was the JAI Pulnix TM-6740GE camera. The camera could record at vector graphics array (640 horizontal $\times 480$ vertical pixels) resolution, capturing up to $200 \mathrm{frames} / \mathrm{s}$. Another decisive feature of the camera was the shutter capability. A high shutter speed is required in order to view a target sports ball as close to static as possible, during flight. The Pulnix camera allowed a maximal electronic shutter speed of $\frac{1}{64000} \mathrm{~s}$. 
The monochrome version was selected ahead of the colour version because of its high signal-to-noise ratio, better contrast, increased resolution, reduced cost, and insusceptibility to chromatic aberration [16]. This camera was capable of providing highly monolithic images suitable for rapid image processing to facilitate fast update speeds within the system. A charge-coupled device (CCD) vision chip was used because of its increased sensitivity.

The physical dimensions of the camera and its housing were such that they permitted compact assembly together with other components. The camera sensor was $3.6 \mathrm{~mm} \times 4.8 \mathrm{~mm}\left(\frac{1}{3} \mathrm{in}\right)$ in size and each pixel was $7.4 \mu \mathrm{m} \times 7.4 \mu \mathrm{m}$ in dimensions.

A C-mount Tamron $6.6 \mathrm{~mm} \times 8.8 \mathrm{~mm}\left(\frac{2}{3}\right.$ in) $25 \mathrm{~mm}$ $f / 1.4$ model lens was used, which allowed an FOV of $32.6 \mathrm{~cm} \times 24.5 \mathrm{~cm}$ at a $1.7 \mathrm{~m}$ stand-off distance, according to

$$
O_{\mathrm{S}}=\frac{W_{\mathrm{D}} I_{\mathrm{S}}}{f_{\mathrm{L}}}
$$

where

$$
\begin{aligned}
& O_{\mathrm{S}}=\text { object size }(\mathrm{mm}) \\
& W_{\mathrm{D}}=\text { working distance }(\mathrm{mm}) \\
& f_{\mathrm{L}}=\text { focal length }(\mathrm{mm}) \\
& I_{\mathrm{S}}=\text { image sensor size }(\mathrm{mm})
\end{aligned}
$$

This ensured that the camera FOV was small at the $x$ and $y$ mirrors, $1.4 \mathrm{~cm} \times 1.9 \mathrm{~cm}$ and $1.9 \times 2.5 \mathrm{~cm}$ at their $10 \mathrm{~cm}$ and $13 \mathrm{~cm}$ stand-off distances respectively. This allowed the camera to 'see' through the mirrors. The lens allowed manual adjustment of the focus and aperture, with locking screws to fix the settings.

\subsubsection{The laser}

A $532 \mathrm{~nm}$ wavelength diode-pumped solid state (DPSS) laser was used to provide $300 \mathrm{~mW}$ of continuous-wave (CW) light. The LTS set-up required the laser only for illumination purposes; therefore, a relatively inexpensive laser could be utilized.

The wavelength chosen for the laser was influenced by two main factors: first, the quantum efficiency; second, the cost. The quantum efficiency is defined as the percentage of photons hitting a photoreactive surface that will produce an electronhole pair. Traditionally, lasers have been manufactured at red wavelengths because of the simplicity of generating laser light at this wavelength; as a consequence the cheapest lasers found on the market fall into this category. The quantum efficiency of the Pulnix camera at red wavelengths was approximately half the quantum efficiencies at blue and green wavelengths of light. Generally, with CCD or complementary metal-oxide-semiconductor cameras the
Table 1 Cost comparison of equal-power DPSS laser sources [18]

\begin{tabular}{lc}
\hline Laser source & Cost (\%) \\
\hline $100 \mathrm{~mW}$ DPSS, 660 nm (red) & 9 \\
$100 \mathrm{~mW}$ DPSS, 532 nm (green) & 22 \\
$100 \mathrm{~mW}$ DPSS, 473 nm (blue) & 69 \\
\hline
\end{tabular}

quantum efficiency has been reported to be a maximum at about $550 \mathrm{~nm}$ [17]. The quantum efficiencies at blue and green wavelengths were very similar for the Pulnix camera; however, with current laser technology, blue light cannot be generated from a cheap laser diode, since complex and expensive optics are required. Table 1 displays a cost comparison of identical-power DPSS lasers, available through a national supplier. Taking into account all the factors the green laser diode was deemed the most suitable for the task.

The laser emitted a collimated beam with a beam diameter less than $1.5 \mathrm{~mm}$ at its source and a divergence of less than $1.5 \mathrm{mrad}$. The laser was mounted within a completely self-contained unit which was thermoelectrically cooled to allow the laser to operate in CW mode at temperatures between $10^{\circ} \mathrm{C}$ and $30^{\circ} \mathrm{C}$.

\subsubsection{Interference filter}

Image-processing tasks, such as blob analysis and the Hough transforms used by Neilson et al. [5], are often complicated by changing background conditions or by having similarly shaped objects in the camera FOV that the camera is trying to detect. Therefore, in order to make this system adaptive to changing ambient lighting conditions, an interference filter was positioned in front of the camera lens. The filter eliminated the intensity of unwanted light, allowing the image-processing task to be carried out extremely effectively, based only on the selected wavelength of laser light in the camera FOV. The Pulnix camera could image visible and infrared light in the range $375-1000 \mathrm{~nm}$; by incorporation of the filter the system was able to detect light only in the range $530 \pm 5 \mathrm{~nm}$ (to match the laser wavelength utilized in section 1.2.2).

The interference filter simplified the image-processing task vastly, since only the projected laser pattern was imaged in a monolithic form, allowing quick and simple image processing, as illustrated in Fig. 2.

\subsubsection{Diffractive optical element}

A diffractive optical element (DOE) is a passive component which allows an input laser beam to be modified into a desired beam shape. The component 
(a)

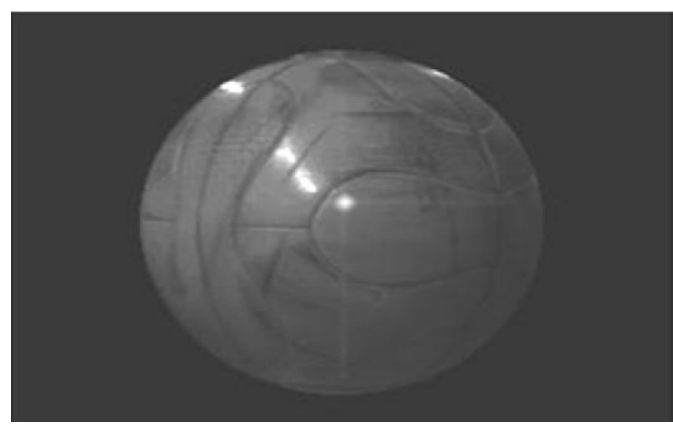

(b)

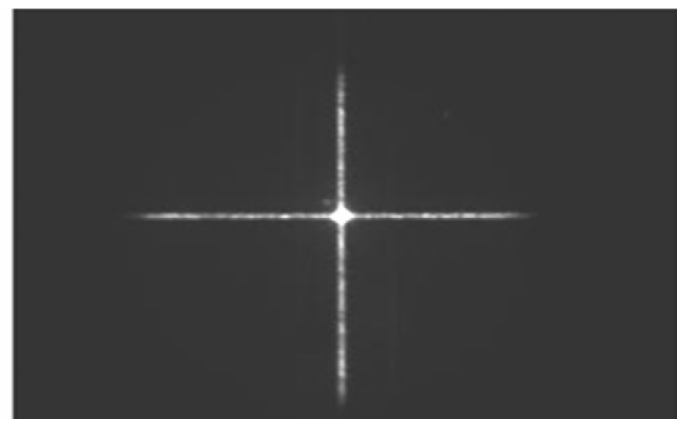

Fig. 2 Pulnix camera snapshots: (a) standard image capture; (b) monolithic image capture using an interference filter $(\lambda=530 \mathrm{~nm})$ in front of the lens

uses the principle of diffraction to modify light of a specific wavelength. Complex illumination patterns can be generated, which would otherwise require complex optical set-ups.

The industry for DOEs, also known as computergenerated holograms, is a relatively new and specialist field. Worldwide there are few manufacturers and the prices of these parts fluctuate greatly. The DOE for the tracking application was designed to emit a 'cross' pattern within a divergence of $25^{\circ}$. Owing to the nature of DOEs, the undiffracted light or 'zeroth order' contains the largest irradiance of light. It is this zeroth order that determines the safety classification which, for practical reasons, was decided to be the eye-safe class $2 \mathrm{M}$ whereby the maximum permissible exposure must be less than $25.4 \mathrm{~W} / \mathrm{m}^{2}[\mathbf{1 9}]$.

\subsubsection{Beam splitter}

In order to align the camera and laser, they must be perpendicular to each other, facing an identical beam splitter mirror which is set at a $45^{\circ}$ angle to both. A plate beam splitter with a 50:50 ratio of reflected to transmitted light was used. This was preferred to a cube beam splitter since it did not distort the camera FOV. The major drawback with the beam splitter arrangement was that only approximately 25 per cent of the original laser light was detected at the camera sensor, owing to the dual-pass design.

\subsubsection{The scanning system}

The scanning system consisted of galvanometers which had three main components: the mirrors, the galvanometers, and the servo controllers. This galvanometer set-up was chosen as the only dynamic part of the LTS and allowed the laser beam to be steered precisely towards the target. The galvanometer system was chosen because of the speed and accuracy with which the mirrors could be moved at a reasonable cost.
The mirrors were required to steer the laser and camera axis towards the ball being tracked, quickly with high resolution. The mirrors were also required to direct the entire laser illumination pattern projected from the DOE; therefore, the larger the mirror aperture, the greater is the potential stand-off distance between the DOE and the scanning mirrors. The mirrors were chosen with a $30 \mathrm{~mm}$ clear aperture made from relatively inexpensive fused silica.

The galvanometer consisted of three parts: first, the actuator that manipulated the mirror load; second, a position detector that fed back the mirror position to the servo controllers; third, the mirror. The galvanometer allowed a high torque to be generated at the mirrors.

Each galvanometer required a servo driver controller, which took the form of an efficient closedloop system that demodulated the current output from the position detector, performed a comparison between this signal and the commanded position signal, and adjusted the actuator until the signals were unity. The controllers purchased were tuned for speed and optimized for large input signals, allowing 20 mrad resolution of the mirror position while permitting the mirrors to scan the entire range of motion $\left( \pm 20^{\circ}\right)$ in $13 \mathrm{~ms}$.

\subsubsection{Operating system}

The LTS was controlled through a PC. The PC was installed with $3.25 \mathrm{~Gb}$ of random-access memory and consisted of a $2.8 \mathrm{GHz}$ dual-core Zeon processor, operating a 32 bit PCI bus functioning in the Windows XP environment.

\subsection{Software}

The main criterion for the software was the ability to create a real-time image-processing platform.

The software purchased to perform the image processing was Common Vision Blox (CVB) developed by Stemmer Imaging. CVB is a modular software 
(a)

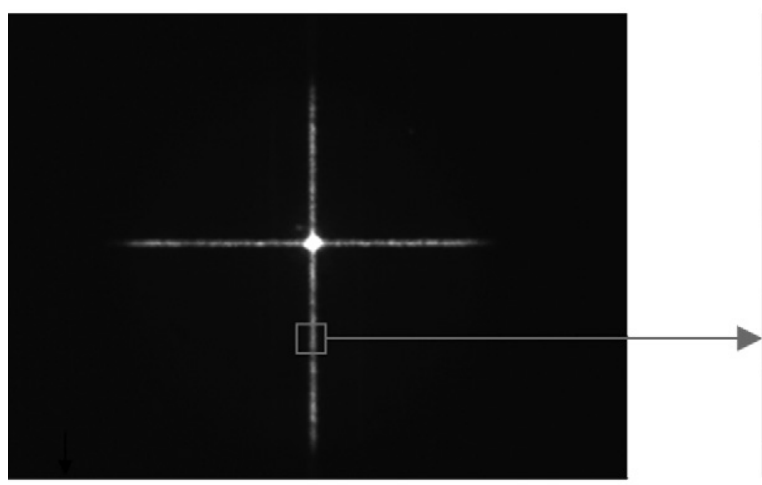

(c) (b)

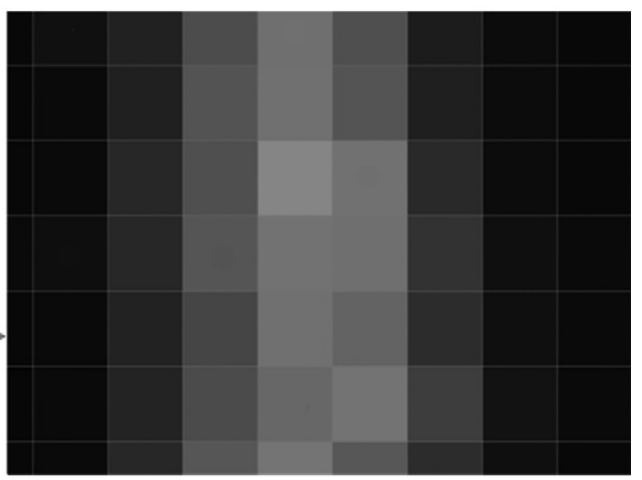

(d)

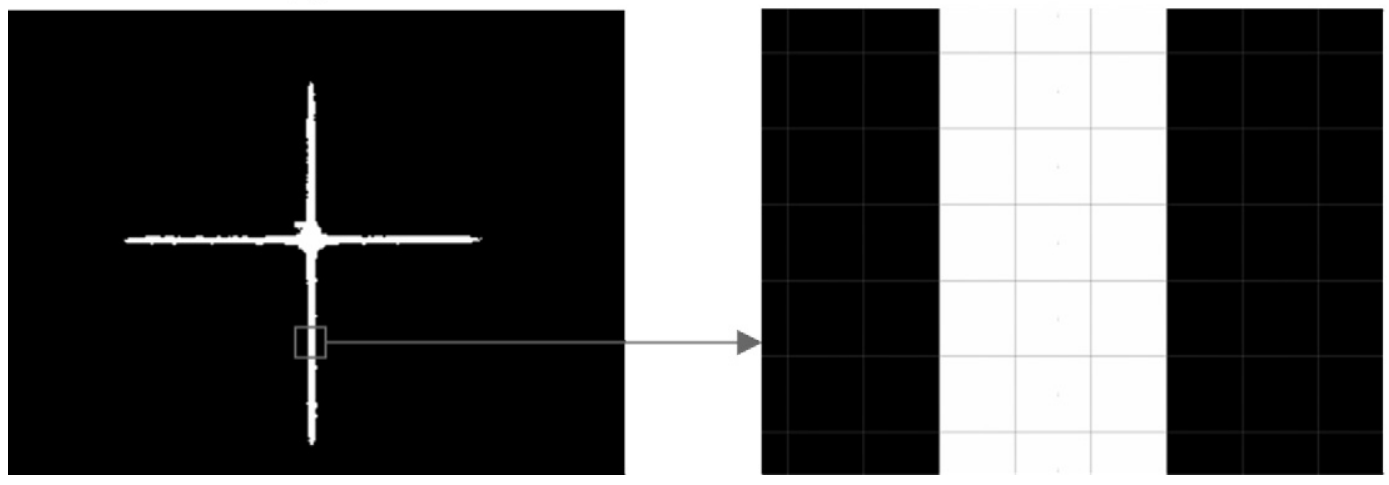

Fig. 3 Image processing: (a) acquired 8-bit image; (b) zoomed-in section showing the grey-scale pixels; (c) image after the LUT processing stage; (d) zoomed-in section showing binary pixels

platform for development and implementation of image-processing tasks with real-time capability, which supported the chosen Pulnix TM-6740GE camera. CVB allowed all the image-processing tools to be implemented as dynamically linked libraries (DLLs), which allowed the integration with other software.

The software used to run the CVB image-processing tools was the 2003 version of Microsoft Visual Studio.NET. The language neutrality of the .NET framework was achieved via a common languages specification. As the framework was designed to work with DLLs, it was straightforward to add the CVB image-processing tools into the Visual Basic (VB).NET code. The .NET framework was designed to run efficiently in Windows, which allowed for a realtime software platform. The programming was completed using VB.NET, which allowed all the necessary algorithms for the operation of the LTS.

\section{TRACKING APPLICATION}

This section reports the image-processing algorithms required to make the system track in real time, the calibration protocol used to make the system work efficiently, a practical example of the system acting as a launch monitor, and a brief validation of the launch conditions measured by the LTS.

\subsection{Image processing}

The image-processing stage was reliant on the fact that the laser and the camera have identical optical axes. The raw 8-bit image captured by the camera, as shown in Figs 3(a) and (b) was transferred directly into a look-up table (LUT) algorithm whereby a fixed threshold value was used to create a binary image, as shown in Figs 3(c) and (d). The LUT allowed predefined values stored in memory to be retrieved extremely quickly. This fixed threshold value was determined in a brief calibration stage, which had to occur only once prior to testing.

The binary image was then processed using a low-pass filter. This was carried out since the laser cross appeared slightly speckled in nature because the laser light struck optically 'rough' surfaces. Therefore, the cross was filtered into a 'smooth' binary shape.

Next, the laser cross was analysed in four individual 'edge' detect algorithms, where each line projecting from the centre was analysed separately. The 'edge' detect algorithm was carried out along the long axis of each line; the intersection point of each 
(a)

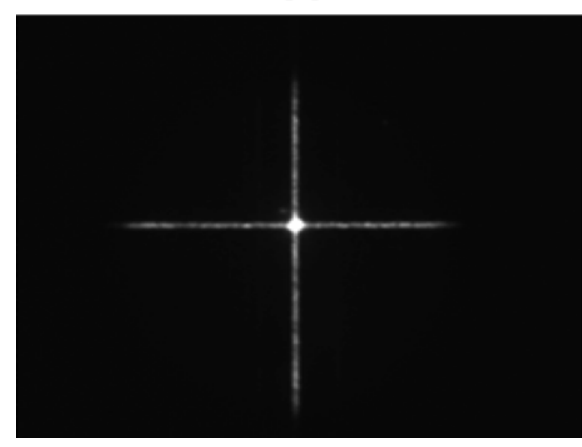

(b)

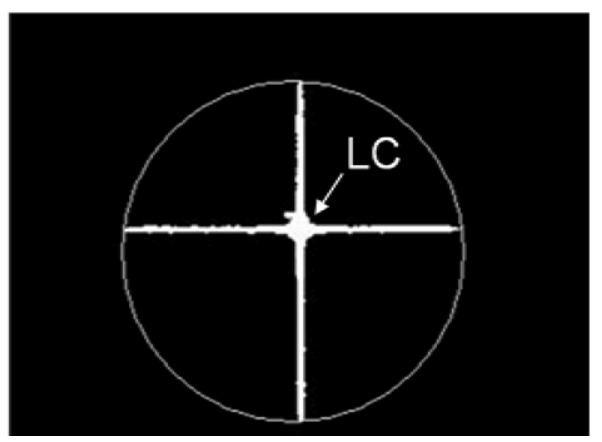

(c)

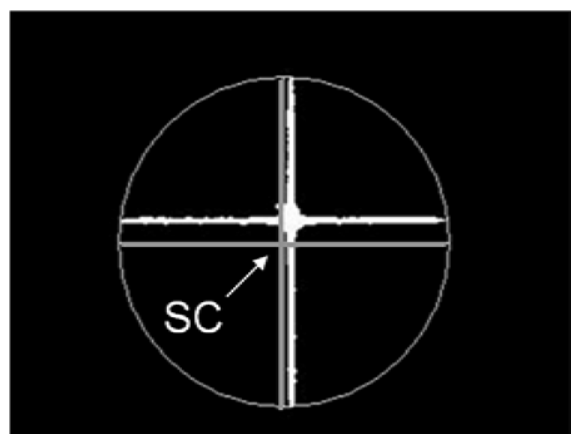

Fig. 4 Summary of image processing: (a) acquired 8-bit image; (b) 'edge' detect algorithm located the edge of the ball (the laser centre point (LC) is in the centre of the camera FOV); (c) the sphere centre point (SC) is calculated

line was located and measured to subpixel accuracy. The four intersection points were defined as the intersection points between the arms of the cross and the perimeter of the ball. The midway points between the intersection points of the horizontal and vertical lines were calculated separately; the two midway points defined the centre point of the sphere in the horizontal direction and vertical direction. The summary of the key image-processing stages is highlighted in Fig. 4.

\subsection{Tracking process}

Once the LTS was set to track the target, the tracking process occurred in a continuous feedback loop and the result of the image-processing stage determined whether the tracking galvanometers had to be repositioned in order to track the ball. When the measured centre point of the sphere was found not to coincide with the centre pixel of the camera, a voltage was applied to the galvanometer mirrors to reposition them, in order to realign the centre point of the sphere with the centre point of the camera sensor array.

In order for the system to track in real time, all the tracking process events as shown in Fig. 5 had to occur rapidly in series.

\subsection{Calibration}

One of the most important parameters within the LTS was the scale factor used to relate the required pixel displacement with the voltage applied to the galvanometer mirrors. If the scale factor was too large, the system would fail to remain fixed on the centre of a static ball, causing a circular saccade around the centre of the ball. Consequently, if the scale factor was too small, the maximum target velocity that the system could track would be reduced accordingly.
Two different approaches were used to achieve the scale factor: a fixed scale factor and an adaptive scale factor. This paper will report the fixed scale factor since the system was found to operate quickly and efficiently using this. Further work is required for the adaptive scale factor.

\subsection{Fixed scale factor}

The fixed scale factor was computed in two stages. In the first stage a target of known diameter was imaged within the camera FOV at a given stand-off distance. From software-based image interrogation a ratio of pixels per millimetre could be established for each of the $x$ and $y$ axes of the camera array.

The second stage was required to relate the voltage applied to the galvanometer mirrors to the corresponding linear movement of the central laser spot at the same stand-off distance. This was achieved using a similar method as used in the first stage, by positioning a target of known diameter within the FOV. Because of the physical offset between the orthogonally aligned galvanometer mirrors along the laser beam path, the ratio for adjustment in the vertical axis was found to be different from that in the horizontal axis. By combining both stages of the computation for the scale factor, a relationship between the galvanometer mirror voltage and imaged pixel dimension was established. This enabled the system to calculate and apply an appropriate voltage to the galvanometers in order to realign the centre point of the sphere with the centre point of the camera sensor array. Differences in both the $x$-axis and $y$-axis scale factors, together with a difference between the ranges in the two directions, were included within the system.

\subsection{Soccer ball tracking}

This section illustrates the system acting as a launch monitor for a player striking a soccer ball. During 


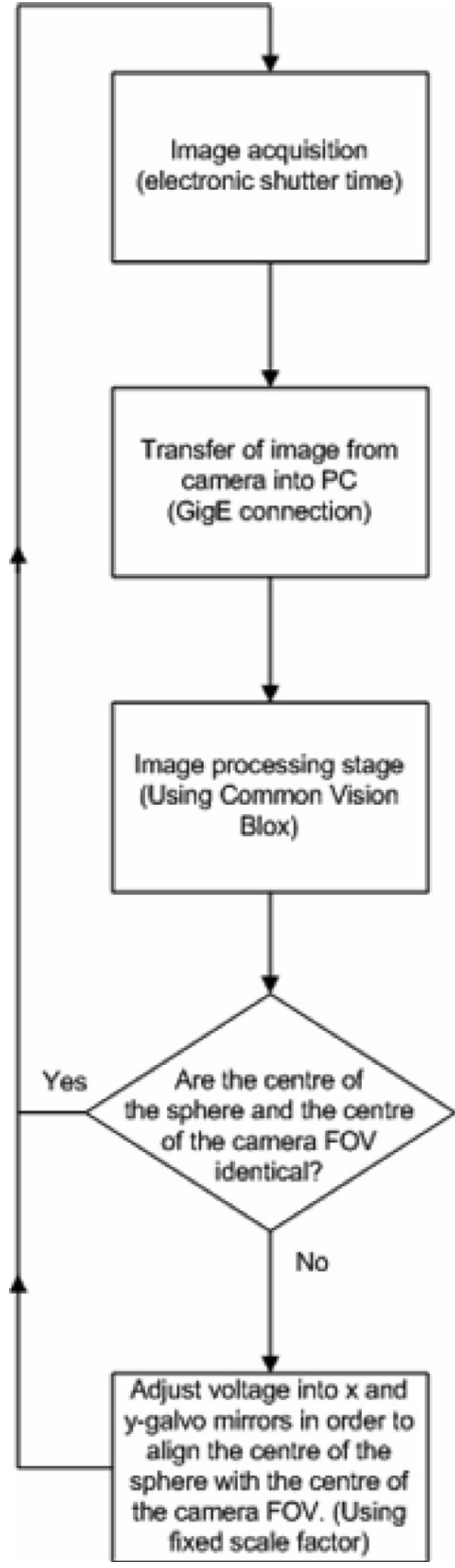

Fig. 5 Order of events in the real-time tracking process

all testing, the output laser beam complied with the level 2M laser safety classification [19].

Owing to the design of the system the envelope within which the tracking is possible is directly dependent upon the stand-off distance between the ball and the LTS. The testing was carried out indoors, with an allowable stand-off distance of $1.7 \mathrm{~m}$ between the ball and system. The larger the stand-off distance between the system and the ball, the larger is the envelope within which tracking is possible. With this set-up the ball could be tracked moving arbitrarily in a space envelope of $1.15 \mathrm{~m} \times 1.04 \mathrm{~m}$. The ball could also be displaced out of plane by $\pm 0.5 \mathrm{~m}$ while the LTS was still able to keep track of the ball.

The time required to process each captured image and to update the galvanometer servo controllers was less than $4 \mathrm{~ms}$. This update speed was operating at the limits of the outlined system.

Given the described capability of the system to track a ball in space with time, it followed that the information gathered by the system could be used to output the launch conditions of a kick, as shown in Fig. 6. In order to act as a launch monitor the LTS had to be manually positioned with its beam incident on the ball before tracking was initiated. The LTS would wait indefinitely for the ball to be kicked, as shown in Fig. 6(a). Once the ball was kicked and measured to have moved more than half a ball diameter in the horizontal axis, as shown in Fig. 6(b), the system was programmed to record each $x$-mirror voltage and $y$-mirror voltage. The mirror voltages were recorded until the ball approached the limit of the system FOV. Once the ball reached the limit of the system FOV, tracking was halted, as shown in Fig. 6(c). Each of the recorded $x$-mirror voltages and $y$-mirror voltages was analysed in order to determine the launch velocity and launch angle, throughout the recording phase. The calculations undertaken within the system were based on an assumption that the ball travelled perpendicular to the LTS; therefore, two-dimensional launch measurements were computed.

The system required the ball to travel orthogonally to the LTS and it was assumed that the ball travelled linearly during the measurement period; therefore, no smoothing functions were carried out. However, the captured data points were divided by two in order to obtain an average point for the ball at the start and end of the measurement period; using these two mean data points, trigonometry and laws-of-motion equations were used to calculate the velocity and launch angle of the ball. The purpose of using mean values was to ensure that noise due to successive data points did not contaminate the data. The launch parameters were displayed immediately after the kick within the LTS software graphical user interface. The measured launch parameters for the kick shown in Fig. 6 were calculated as $9.1 \mathrm{~m} / \mathrm{s}$ velocity and $18.7^{\circ}$ launch angle. 
(a)

(b)

(c)

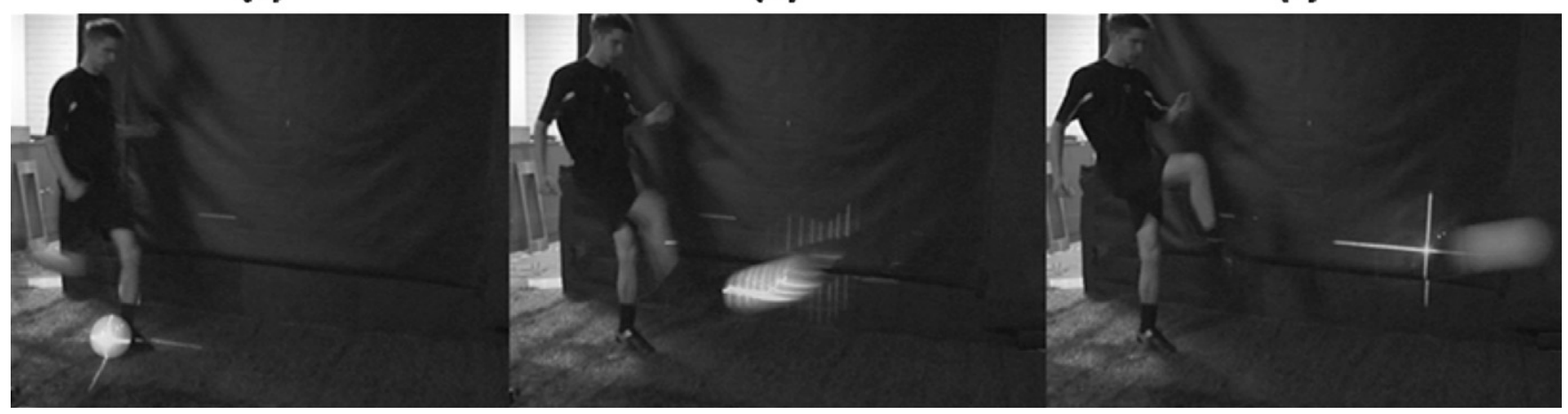

Fig. 6 Launch monitor in action

\subsection{Validation of soccer ball tracking}

The validation stage was carried out indoors and split into two: velocity and launch angle measurement. In order to recreate a realistic soccer strike scenario a purpose-built mechanical kicking simulator [20] was utilized to ensure repeatable strikes at a set velocity in a similar scenario to an actual player striking the ball. The LTS system was set up perpendicular $1.7 \mathrm{~m}$ from the line of ball travel. Operating concurrently to the LTS system was a high-speed video (HSV) camera (Photron SA1.1 250 FPS 1/1000 shutter speed) set up perpendicular $2 \mathrm{~m}$ from the line of ball travel. The ball was to be struck initially at a low velocity and then increased until the LTS could no longer track the ball. In order to understand the repeatability of measurement, each strike velocity was repeated three times.

The LTS carried out all the computation internally to output the launch velocity and launch angle of the kicks. The HSV capture data were manually digitized (Image Pro Plus) to output the launch velocity, which was computed over the same distance as the LTS was able to track the soccer ball. The diameter of the ball was used as the reference dimension in the HSV camera FOV.

Figure 7 shows the results for the velocity measurements. A discrepancy of between $0.11 \mathrm{~m} / \mathrm{s}$ and $1.3 \mathrm{~m} / \mathrm{s}$ was found between the LTS and the HSV data, with the LTS measuring the velocity of the ball to be higher by an average of $0.8 \mathrm{~m} / \mathrm{s}$ throughout the velocity range from $5 \mathrm{~m} / \mathrm{s}$ to $10 \mathrm{~m} / \mathrm{s}$. It is therefore thought that a systematic error occurred throughout capture and/or analysis of the launch data and was most probably attributed to misalignments of either the LTS or the HSV set-up.

In order to validate the launch angle measurement a platform was constructed that could be tilted from $0^{\circ}$ to $45^{\circ}$ with an accuracy of $\pm 0.05^{\circ}$. A digital inclinometer (SmartTool) was used to report the angle at which the platform was fixed. This allowed the ball to be rolled along the platform, recreating realistic

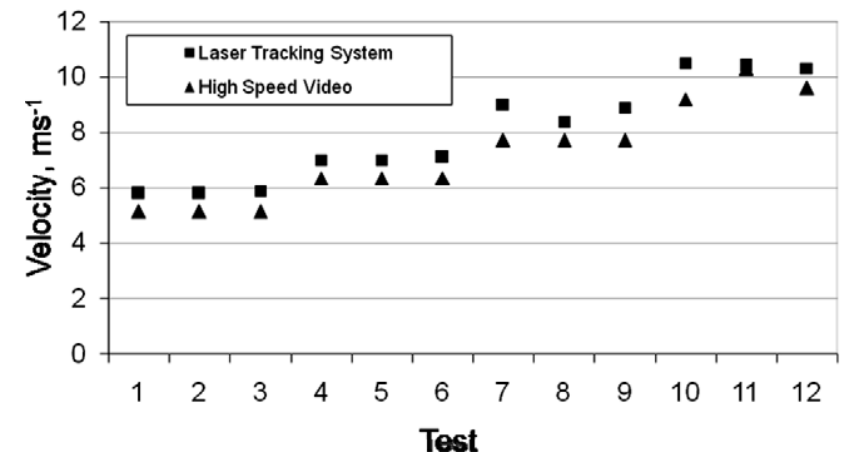

Fig. 7 Output velocity values for the LTS and HSV

Table 2 Measured angles for the LTS and digital inclinometer

\begin{tabular}{rcc}
\hline Test & $\begin{array}{l}\text { Laser tracking } \\
\text { system (deg) }\end{array}$ & $\begin{array}{l}\text { Inclinometer } \\
\text { (deg) }\end{array}$ \\
\hline 1 & 5.2 & 4.9 \\
2 & 5.1 & 4.9 \\
3 & 5.2 & 4.9 \\
4 & 9.9 & 9.9 \\
5 & 10 & 9.9 \\
6 & 9.9 & 9.9 \\
7 & 14.9 & 15.1 \\
8 & 14.8 & 15.1 \\
9 & 14.8 & 15.1 \\
10 & 19.4 & 19.9 \\
11 & 19.4 & 19.9 \\
12 & 19.4 & 19.9 \\
\hline
\end{tabular}

launch angles for a soccer kick. Four different matchrealistic angles of launch were tested towards the centre of the LTS FOV. In order to gain an understanding of repeatability of the system, measurements at each angle were repeated three times.

Table 2 shows the results for the launch angle validation test. Throughout testing, the LTS was demonstrated to record launch angle measurements to within $0^{\circ}$ to $\pm 0.5^{\circ}$ of those measured by the inclinometer. However, at smaller launch angles the LTS was found to record a larger angle than the 


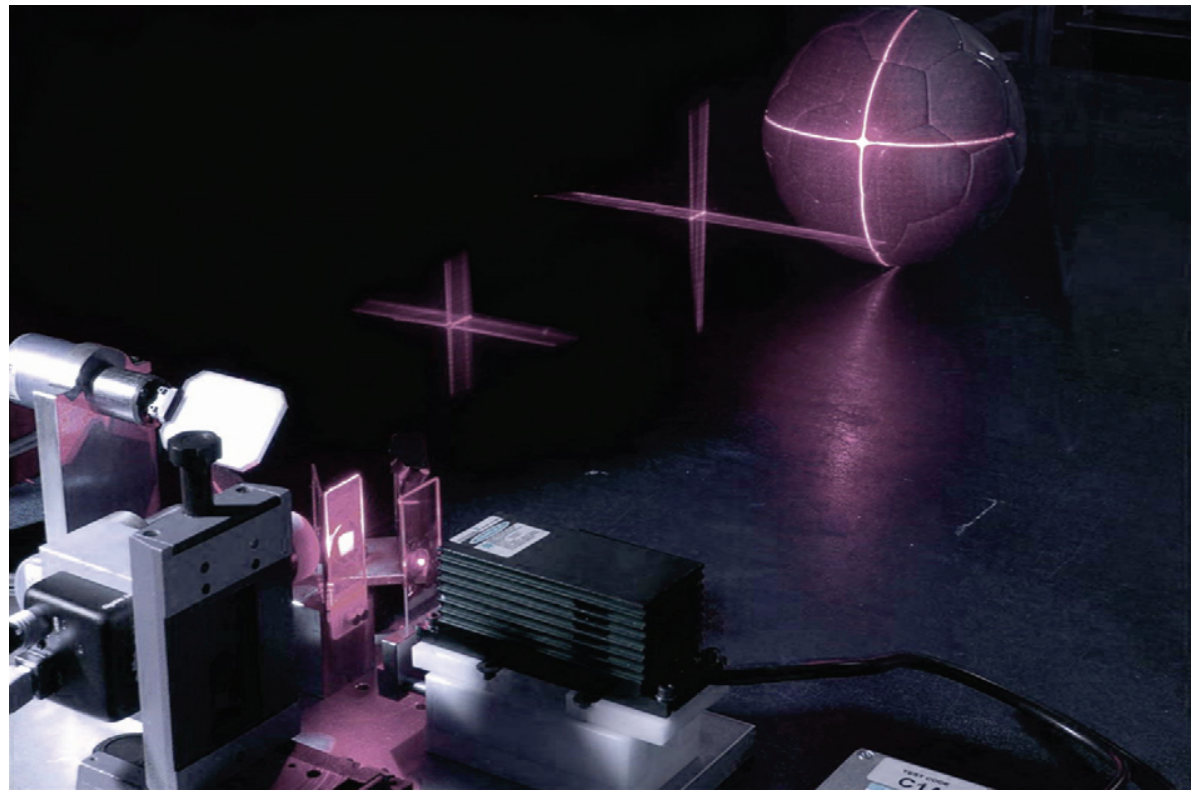

Fig. 8 The LTS

inclinometer with the converse occurring at larger angles. This discrepancy is thought to be dependent upon where in the FOV the ball is measured.

\section{SOURCES OF ERROR}

The development of the LTS is considered to be an advancement of current laser tracking methods; however, the current system does have some limitations. The accuracy of the velocity and launch angle measurement are considered to be in good agreement with competing systems at this stage and the technology is considered worth pursuing in further work by addressing the following sources of error.

The current system currently utilizes a fixed scale factor to control the adjustment of the $x$-galvanometer mirror and $y$-galvanometer mirror. A more advanced (adaptive) scale factor would facilitate a more precise adjustment which would result in the derivation of a more accurate trajectory measurement from the recorded mirror voltages. This in turn would allow more accurate velocity and launch angle measurements. The downside of utilizing an adaptive scale factor would be that drastically more (greater than 2.5 times) central processor unit time would be required to analyse the acquired images.

Because of the optical nature of the system, the colour (graphics) on the ball would ideally be monochromatic, since this would allow the fixed threshold setting for image processing to be set accordingly. Certain graphics on the surface of the ball were found to affect the systems ability to track the ball in motion reliably. Further work is required to develop image-processing protocols capable of resolving issues related to ball graphics.

\section{CONCLUSIONS}

An LTS was designed, developed, and demonstrated to track non-marked soccer balls continuously in a non-contact approach, i.e. tracking the motion of a ball in real time up to approximately $10 \mathrm{~m} / \mathrm{s}$ within a stated volume. Implemented hardware and software choices have been outlined, for the successful operation of the device. The concept behind the tracking principle was clearly explained; Fig. 8 displays a photographic image of the device set-up to track a ball.

The LTS was demonstrated to be capable of tracking soccer balls moving at higher speeds than any other similar laser-based device previously reported. The prototype system was demonstrated to function in a range of ambient lighting conditions.

The application of the LTS has been demonstrated in this paper using a soccer ball. It is envisaged that the current technique could be applied to any sports ball or spherical object.

While the principles outlined in this paper provide the basis for a functional tracking system, it is likely that electronic and computing technology will continue to advance. The current system has been developed and demonstrated to operate in the Windows environment on a PC (circa 2005). However, if the image processing were to be carried out on specifically designed digital signal-processing boards or field-programmable gate arrays in future, an increase in image-processing and update speeds 
could be anticipated, allowing the real-time component of the system to operate even more quickly, thus enabling the LTS to track faster-moving objects.

\section{ACKNOWLEDGEMENTS}

The authors wish to thank the Engineering and Physical Sciences Research Council for funding this work and Mr A. Hallam and Mr S. McLeod for their help and input to the investigation.

(C) Authors 2010

\section{REFERENCES}

1 SGMA, Manufacturers sales by category report, US wholesale value of annual manufacturers' shipments (\$millions), 2008, available from http://www.sgma. com/reports/219_Manufacturer-Sales-by-Category2008 (accessed 12 December 2008).

2 Tavares, G., Shannon, K., and Melvin, T. Golf ball spin decay model based on radar measurements. In Science and golf III, Proceedings of the World Scientific Congress on Golf, St Andrews, UK, 1998, pp. 464-472 (Human Kinetics, Champaign, Illinois).

3 Hawkins, P. and Sherry, D. Video processor systems for ball tracking in ball games. World Wide Pat. WO01/41884A1, Int. Pat. Applic. A63B71/06, 14 June 2001.

4 Johnson, H. and Foster, M. C. Golf ball tracking device. World Wide Pat. WO03032006, 17 April 2003.

5 Neilson, P., Jones, R., Kerr, D., and Sumpter, C. An image recognition system for the measurement of soccer ball spin characteristics. Measmt Sci. Technol., 2004, 15, 2239-2247.

6 Tuxen, F. A method of and an apparatus for determining information relating to a projectile, such as golf ball. World Wide Pat. WO2005116678, 8 December 2005.

7 Durst, F., Lehman, B., and Tropea, C. Laser-Doppler system for rapid scanning of flow fields. Rev. Scient. Instrum., 1981, 52(11), 1676-1681.

8 Stanbridge A. and Ewins, D. Modal testing of rotating discs using a scanning laser Doppler vibrometer. In Proceedings of the 1995 Design Engineering Technical Conferences, 1995, vol. 3B, pp. 1207-1213 (ASME International, New York).

9 Castellini, P. and Tomasini, E. Novel image tracking laser Doppler vibrometer for application on automotive field. In Proceedings of the 20th International Modal Analysis Conference on Structural dynamics (IMAC-XX), Proceedings of SPIE, vol. 4753, Los Angeles, California, USA, 4-7 February 2002, pp. 1004-1008 (SPIE, Bellingham, Washington).

10 Perrin, S., Cassinelli, A., and Ishikawa, M. Laser-based finger tracking system suitable for MOEMS integration. In Proceedings of Image and vision computing (IVCNZ 2003), Palmerston North, New Zealand, 26-28 November 2003, pp. 131-136 (Institute of Information Sciences and Technology, Massey University, Palmerston North).
11 Perrin, S., Cassinelli, A., and Ishikawa, M. Gesture recognition using laser-based tracking system. In Proceedings of the Sixth IEEE International Conference on Automatic face and gesture recognition, Seoul, Republic of Korea, 17-19 May 2004, pp. 541-546 (IEEE, New York).

12 Cassinelli, A., Perrin, S., and Ishikawa, M. Smart laser-scanner for 3D human-machine interface. In Proceedings of the ACM SIGHI International Conference on Human factors in computing systems, Portland, Oregon, USA, 2-7 April 2005, pp. 33-35 (ACM, New York).

13 Wilson, A. Product focus - PCI express captures framegrabber designs. Vision Systems Des., 2006, 11(11), 95-101.

14 Williamson, M. Selecting image-transfer techniques for high speed imaging. Europhotonics, October-November 2006, 30-31.

15 Wilson, A. Product focus - standards propel gigabit ethernet to the forefront. Vision Systems Des., 2007, 12(2), 31-38.

16 Dougherty, J. Using filters in colour inspection. Vision Systems Des., 2007, 12(6), 33-38.

17 Wilson, A. Product focus - understanding camera performance specs. Vision Systems Des., 2007, 12(7), 39-45.

18 Scitec Instruments, Lasers, 2009, available from http://www.scitec.uk.com/lasers/ (accessed 7 July 2009).

19 IEC 60825-1 ed2.0 Safety of laser products - Part I: equipment classification and requirements, 30 March 2007 (International Electrotechnical Commission, Geneva).

20 Ronkainen, J., Holmes, C., Harland, A., and Jones, R. A comparative study of ball launch measurement systems; soccer case study. Engng Sport, 2008, 7, 239-246.

\section{APPENDIX}

\section{Notation}

$\begin{array}{ll}\text { CCD } & \text { charge-coupled device } \\ \text { CVB } & \text { Common Vision Blox } \\ \text { CW } & \text { continuous wave } \\ \text { DLL } & \text { dynamically linked library } \\ \text { DOE } & \text { diffractive optical element } \\ \text { DPSS } & \text { diode-pumped solid state } \\ f_{\mathrm{L}} & \text { focal length (mm) } \\ \text { FOV } & \text { field of view } \\ \text { GigE } & \text { gigabit Ethernet } \\ \text { HSV } & \text { high-speed video } \\ I_{\mathrm{S}} & \text { image sensor size (mm) } \\ \text { LC } & \text { laser centre point } \\ \text { LTS } & \text { laser tracking system } \\ \text { LUT } & \text { look-up table } \\ O_{S} & \text { object size (mm) } \\ \text { PC } & \text { personal computer } \\ \text { PCI } & \text { peripheral component interconnect } \\ \text { SP } & \text { sphere centre point } \\ \text { VB } & \text { Visual Basic } \\ W_{\mathrm{D}} & \text { working distance (mm) }\end{array}$

